\title{
The Issues of Electronic Equipment Grounding at the Power Facilities
}

\author{
Vladimir Gurevich, Ph.D. \\ Central Electrical Laboratory Israel Electric Corp. Haifa, Israel
}

\begin{abstract}
Grounding of electronic equipment at industrial and power facilities is usually perceived as a prerequisite to ensure equipment survival under electromagnetic interference. Is it really true? Do common approaches to electric equipment grounding really solve the problems of electromagnetic compatibility? The fact is that the answer to this question is dubious and the current methods of grounding are controversial in their essence. I suggest a different approach to electronic equipment grounding that will allow avoiding the existing controversies.
\end{abstract}

Keywords: grounding, electronic equipment, electromagnetic interferences, floating grounding, EMP, HEMP, filters

\author{
"Grounding may not be the solution; \\ rather, it could be part of the problem" \\ Joffe E. B., Lock K. S.
}

(Grounds for Grounding. A circuit-to-System Handbook)

\section{INTRODUCTION}

According to the regulatory documents, there are two types of grounding: protective (for electric safety) and functional (for electric installation operability, but not for electric safety). Modern control, protection, and automation electronic devices used in power industry are equipped with internal electronic circuits fully isolated from all external circuits. For example, digital protective relays (DPR) have internal current and voltage transformers built into their analogue input circuits. These transformers are intended to isolate the internal analogue-to-digital converters from external current and voltage circuits. The logical inputs of DPR are made with optocouplers that efficiently insulate the inputs from the outputs. The output circuits of DPR are built on electro-mechanic relays that provide insulation of the internal electronic circuit from external circuits. The DPR internal switching mode power supply contains a transformer that provides galvanic insulation of the circuit's internal elements from external supply circuits. Moreover, this direct current external supply circuit is generally fully insulated from the earth. Thus, on the one hand, modern electronic devices used in power systems do not require functional grounding for their operation. However, on the other hand, grounding of electronic equipment is often perceived as the main measure to protect the equipmentagainstelectromagnetic interference. In other words, grounding is usually deemed as a key measureto ensure efficient performance of equipmentunder the real-life electromagnetic interference. It is sufficing to mention the name of the publication [1] that equals electromagnetic compatibility (EMC) with grounding of automation and control systems. According to [2]: "for many decades there have been many huge myths surrounding the word "ground", both in circuit design and in EMC design"

Hence, the issues of electronic equipment grounding require a more thorough investigation.

\section{TUPES OF ELECTROMAGNETIC INTERFERENCES}

Let us discuss the four major types of interference:

- Conducted

- Inductive

- Capacitive

- Electrostatic 
Conducted interference spreads upon direct electric contact between electric circuits. Such interference can be subdivided as follows:

- wire-earth interference -voltage is applied between each conductor and the earth. It is also known as asymmetrical, in-phase or common mode (CM) interference.

- wire-wire interference -voltage is applied between separate electric circuits or between the elements of the same electric circuit. It is also known as symmetrical, out of phase or differential mode (DM) interference.

Unlike DM interference, CM interference usually does not result in electronic equipment faults. However, it can make such equipment inoperative due to electric disruption of internal insulation (or p-n-junctions) in the electronic chips and the microprocessors. On the other hand, when electronic equipment is fully isolated from the earth, pulse interference and voltage spikes to earth (CM interference) cannot affect such equipment. This is similar to the situation when high voltage to earth does not kill the birds sitting on high-voltage wires. The DM interference is not connected to the availability or lack of grounding.

As for inductive interference that spreads through the electromagnetic fields, it is known that efficient protection against this kind of interference is ensured by putting sensitive electronic equipment into the closed metal enclosures (acting as the "Faraday cage"). It is also known that Faraday cage grounding does not affect its ability to attenuate the inductive interference.

The situation with capacitive interference is more complicated, as this kind of interference spreads through the capacitance between the adjacent wires and closely located metal enclosures, as well as the above mentioned elements and the earth. Grounding of wire screens and metal enclosures (same as the capacitance reduction) allows eliminating the CM capacitive interference and has almost no effect on the DM capacitive interference.

Electrostatic interference may appear due to static charge accumulation on the enclosure insulated from the earth and periodic discharges to the earth. Grounding of the enclosure prevents the static charge accumulation. However, in order to eliminate electrostatic (or partially even capacitive) CM interference, low-resistance grounding is not necessary. It is sufficient to connect the enclosure to the grounding system through a high-resistance resistor (about 100MOhm).

Obviously, grounding of the DPR enclosure cannot cure all types of interference that come to the internal electronic elements via the cables, and the wires connected to the DPR. Nonetheless, the internal "ground" of the electronic circuit of many DPR types that acts as a circuit of "zero (reference) potential" is usually connected to the metal enclosure and, consequently, to the external grounding system. Sometimes, the separate protective grounding (signal reference subsystem) is used to protect sensitive electronics. Such a system is connected to the common ground system at one point (which does not really change the idea of grounding). At the same time, it is supposed that various electronic devices interconnected through the data channels and the electrical cables share zero (reference) potential. This prevents the fault of highly sensitive electronic equipment under electromagnetic interference, generating additional difference of potentials between the zero potential circuits, if they are not grounded. This is the approach according to [3]: "provides a reference potential for relaying and other equipment, and serves to protect equipment and personnel from power-frequency disturbances." This is a common approach and practice to ensure the electronic equipment EMC. However, it is mentioned in [4], "many interference problems occur because designers treat the ground as ideal and fail to give proper attention to the actual characteristics of the grounding system."

\section{Chalenges of the Conventional Grounding Systems}

It is known that the grounding system potential to earth can increase upon a lightning strike. At the same time, it is considered that if all the electronic devices share a common potential of the grounding system, i.e. there is no difference in potentials between the reference potential circuits of various devices, and such an increase in common potential and its difference from zero appearing simultaneously in all the devices cannot cause them to fault. The whole theory of grounding is based on this assumption, prescribing to maintain minimum resistance of the grounding system's elements, as well as use of equipotential planes and other measures aimed at preventing the difference of potentials between zero potential circuits distanced from each other (and hence grounded at different

International Journal of Research Studies in Electrical and Electronics Engineering (IJRSEEE) Page | 12 
locations), but interconnected through data channels and electric cables. Regarding this, the processes running in a single electronic device during the potential rise in a zero potential circuit are not considered. Any electronic circuit contains many non-linear elements, as well as capacitance and inductance elements connected to the zero potential circuit. Due to this, the voltage at different points of the circuit does not synchronously fully rise upon the circuit potential spikes. To better understand this process, imagine a plate supporting weights of different mass that are attached to this plate by means of springs of various rigidity. If we lift the plate gradually (i.e. gradually increase the potential energy), the potential energy of all the elements resting on this plate increases simultaneously. However, if we lift the plate abruptly, the elements do not simultaneously change their position and potential energy. In addition, mechanical connections (if any) between such elements can be broken. Thus, the availability of an equipotential plane and the maintaining of zero difference between the zero potential circuits of different devices do not guarantee the absence of faults in highly sensitive electronic equipment. In real life, when electronic devices are located at spacious facilities, it is very difficult and sometimes even impossible to maintain zero difference of potentials between zero potential circuits (i.e. a condition of "lack of even the smallest electric potential on the enclosure" [5]) upon a lightning strike, regardless of all the wiles and appreciation of the grounding system.

According to [6], if there are many electric devices, and separate pieces of electronic equipment are significantly distanced from each other and grounded at their particular locations, the high difference of potentials (up to $10 \mathrm{kV}$ or even more) inevitably appears between the grounding points upon a lightning strike. High voltage between the distanced grounding points occurs due to a voltage dip on the grounding system elements under the lightning currents and short-circuit currents flowing through such elements. According to [7]: "the bigger the area of the protected facility, the higher the problems probability". The basic standard for the electronic equipment's grounding systems [8] says: "Under lightning or power system fault conditions, the potential difference between the electronic load equipment and grounded objects may be sufficient to cause a safety hazard or disrupt electronic load equipment performance."

Based on the above, it can be concluded that the low-resistance grounding of enclosures of most types of modern sensitive control, automation, and protection electronic equipment inevitably increases the probability of fault. This is caused by a lightning current flowing through the grounding system upon ordinary lightning charges. However, lightning charges are not the only sources of powerful electromagnetic impacts on electronic devices.

Recently, the power systems protection against the so-called Intentional Destructive Electromagnetic Impacts (IDEI) [9], especially against such powerful interference as the High Altitude Electromagnetic Pulse of nuclear explosion (HEMP), has become very relevant. In addition to that, the impact of HEMP onto a grounding system is significantly different from that of a lightning discharge [10]: "The EM environment generated by lightning differs from that of HEMP in energy spectral distribution rise time, current levels, pulse repetition and coverage area".

First, lightning discharge is a localized impact on the grounding system, while the E1 component of HEMP creates a pulse of distributed electromagnetic field near the soil surface with the field gradient up to $50 \mathrm{kV} / \mathrm{m}$. This field has both vertical and horizontal components and covers a very large area (hundreds and thousands of square kilometers).

Second, the E1 HEMP parameters (2/50 nanoseconds) significantly differ from those of a lightning pulse (1.2/50 microseconds).

Third, when lightning strikes a lightning rod and the grounding system, we have to reduce the resistance of the grounding system to a minimum in order to decrease the voltage drops on current conducting parts when the lightning current is passing through them. Alternatively, high voltage HEMP pulse affects all the grounding system's elements simultaneously, and they start acting as a huge antenna that absorbs the energy from the vast area. The lower the resistance of the elements in such an antenna, and the bigger the antenna is in size, the higher its absorbing capacity will be and the more energy will be delivered directly to the equipment connected to such an antenna. This refers to impulse voltage with the amplitude of hundreds of kilovolts.

The difference between the lightning and HEMP is also mentioned in other publications. For example, [11] directly states that "a "ground" is commonly thought of as a part of a circuit that has relatively low impedance to the local earth surface. A particular ground arrangement that satisfies this definition may, however, not be optimum and may be worse than no ground for EMP protection"; 
[12]suggests that: "techniques used for protecting equipment from slowly rising lightning transients are not necessarily effective against the fast-rising EMP-induced transients";[13] suggests that: "Ideally, grounding would keep all system components at a common potential. In practice, because of possible inductive loops, capacitive coupling, line and bonding impedances, antenna ringing effects, and other phenomena, large potentials may exist on grounding circuits. The choice of grounding concept is therefore important in the EMP protection philosophy". Unfortunately, this document is limited to the fact statements and offers no special grounding systems that can address the controversy between the need to have a grounding system and the danger that it can cause in case of HEMP impact. The authors of [14] are even more categorical; they make a straightforward suggestion that the grounding system has an adverse effect on the operation of electronic equipment: "If we don't take special measures, both types of grounding systems (internal and external) can become the main source of interferences caused by the HEMP. External grounding devices cannot act as "bypass" for HEMP as the latter is very spacious and deeply penetrates into the soil. Moreover, absence of the loop of external grounding does not exclude a possibility of the HEMP's interferences to occur". A similar conclusion is also made in [10]: "a HEMP can induce very large currents and voltages onto the ground network "antenna". These ground network currents and voltages pose a serious threat of damage to electronic components..."

The inconsistency of the situation is clearly highlighted in the fundamental work [15] on more than 1,000 pages. The title of Section 4.1.1 speaks for itself: "Grounding May Not Be the Solution; Rather, It Could Be Part of the Problem"

In addition, there are two contradictory ideas on page 935 :

- "The primary effect of the HEMP is, therefore, the production of large voltages or currents in large structures and conductors such power lines, buried cables, and antennas, as well as in facility grounding systems"

- "The goal of all grounding and bounding techniques is to redirect the HEMP-induced currents to the earth."

The first idea suggests that the grounding system is a receptive capacity that absorbs HEMP energy, while the second idea states that the grounding system is a source of high voltage that brings HEMP energy to the grounded equipment.

The filters designed to protect sensitive inputs and the power supply circuits of electronic equipment, Fig. 1, represent another grounding-related problem. It is obvious from Fig. 1 that CM asymmetrical filters (also known as single line filters) are simpler than the symmetrical DM filters (also known as two line filters) -they are smaller in size and cheaper, and have fewer components. That is why the majority of manufacturers consider the $\mathrm{CM}$ asymmetrical filters as a preferable means to protect against HEMP. Other types of filters are not even considered in [16], which is devoted to the analysis of various types of filters designed to protect equipment against HEMP.

Fig. 1 shows that such filters weaken the signal delivered to their input due to the introduction of losses by a series of inductors, and shunting of pulse signal to the earth by capacitors. The filter is operative only if the terminal located on the enclosure is connected to the grounding. Since this terminal is not insulated from the enclosure, the enclosure often acts as a common grounding rod, Fig. 2.
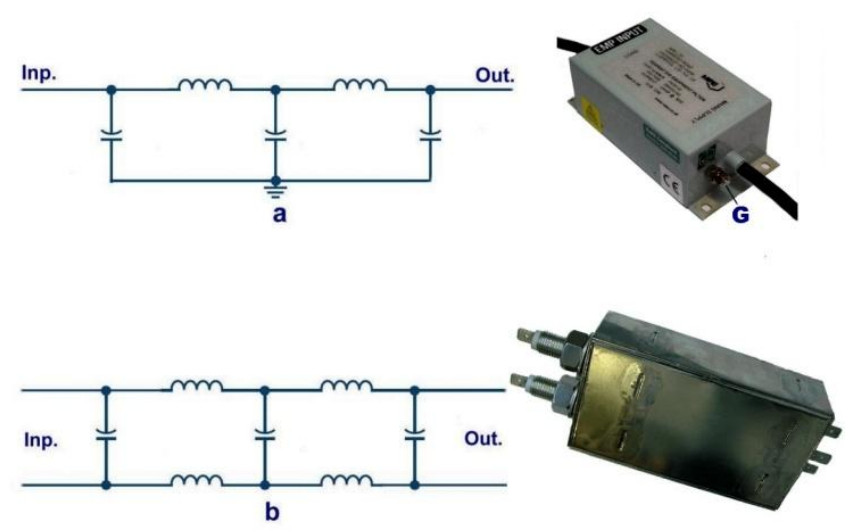

Fig1. Simplified diagrams and appearance of LC-filters intended for protection against HEMP. a asymmetrical common mode filters; $b$ - symmetrical differential mode filters

International Journal of Research Studies in Electrical and Electronics Engineering (IJRSEEE) Page | 14 
Thus, these filters are designed to divert the energy delivered to their inputs to the area of zero potential, which is thought to be the grounding system. In fact, it does not perform in this manner. We can only guess how such a filter will behave when a high potential simultaneously occurs at both its input and a common grounding rod (instead of zero potential). In any event, it will certainly not perform properly under these conditions.
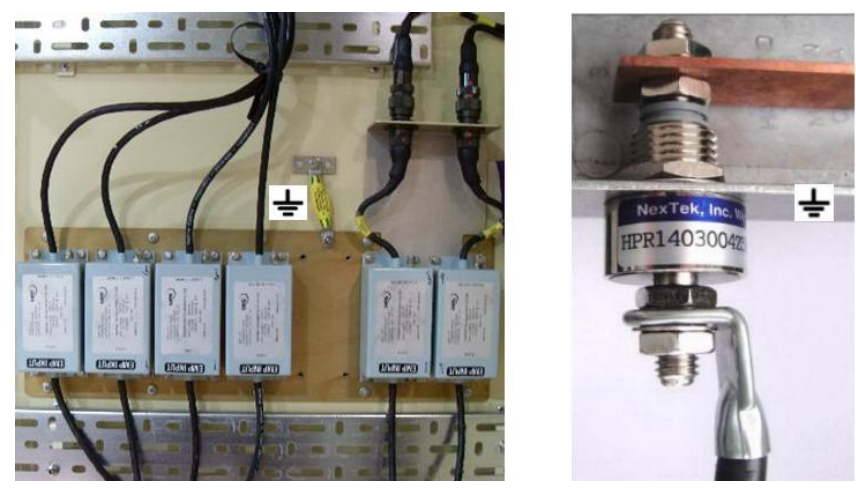

Fig. 2.HEMP filters with enclosure that acts as a grounding electrode.

According to [17], these filters do not always meet acceptable specification and are not recommended as a means of protection against HEMP. Perhaps, due to this, some manufacturers do not mention their type in the technical brochures. Although others do, such specifications are too complicated for a non-specialist in the field of filters, consequently confusing the users. For instance, a brochure of one of the English companies (MPE) provides a description of "two phase" filters (for alternating current) and "two line" filters (for direct current), designed to be used in the circuits insulated from the earth, but the actual specifications given describe the asymmetrical CM filters.

\section{Floating Grounding}

If the grounding causes so many problems, should we not ground the electronic equipment at all? For some reasons, a report of the special working group C4.206 CIGRE discussing the protection of the power system's electronic equipment from Intentional Electromagnetic Impacts [18] does not entirely cover the issue of electronic equipment grounding. This way of grounding (more correctly "nongrounding") is called "floating ground" in the technical literature, e.g. in basic American military standards for grounding systems MIL-HDBK-419A [19], from which it migrated to other military standards and instructions, e.g. [13]. This type of grounding is described in [19 and 13] as follows:

"The effectiveness of floating ground systems depends on their true isolation from other nearby conductors, i.e., to be effective, floating ground systems must really float. In large facilities, it is often difficult to achieve a completely floating system, and even if complete isolation is achieved it is difficult to maintain such a system. In addition, a floating ground system suffers from other limitations. For example, static charge buildup on the isolated signal circuits is likely and may present a shock and a spark hazard. In particular, if the floated system is located near high voltage power lines, static buildup is very likely. Further, in most modern electronic facilities, all external sources of energy such as commercial power sources are referenced to earth grounds. Thus, a danger with the floating system is that power faults to the signal system would cause the entire system to rise to hazardous voltage levels relative to other conductive objects in the facility. Another danger is the threat of flashover between the structure or cabinet and the signal system in the event of a lightning stroke to the facility. Not being conductively coupled together, the structure could be elevated to a voltage high enough relative to the signal ground to cause insulation breakdown and arcing."

Alternatively, the specialists of Associated Power Technologies (APT) - one of the leading manufacturers of powerful power sources -state that [20]: "Grounded systems can present their own set of problems... The dark side to grounding systems is that they can create issues that affect the rest of an electronic system... Small ground loops inject noise onto a system and cause interruption or loss of communication on data lines such as RS-232 or GPIB interfaces. Large ground loops can damage electronic equipment and even pose safety hazards if the ground loop currents are large enough."

Based on the analysis carried out in [20], these specialists conclude that the floating ground is a preferred measure of electronic systems protection, emphasizing that this approach is standard for the products manufactured by their company.

So, is there any way to solve this controversial situation?

International Journal of Research Studies in Electrical and Electronics Engineering (IJRSEEE) Page | 15 


\section{Solution: SPEcial FloAting Ground}

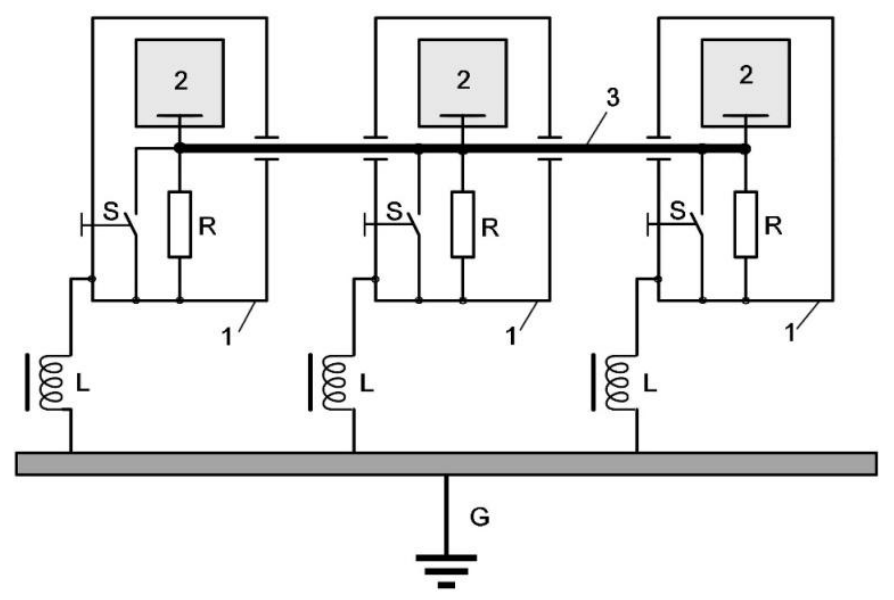

Fig3. Suggested grounding system: special floating ground1 - metal cabinets with electronic equipment; 2 electronic devices; 3 - copper bus establishing the local area of common (reference) zero potential (local ground); $R$ - high-resistance high voltage resistors (100MOhm); $S$-contact-breaker with electric locker on the cabinet's door; L-choke; $G$-facility's grounding system
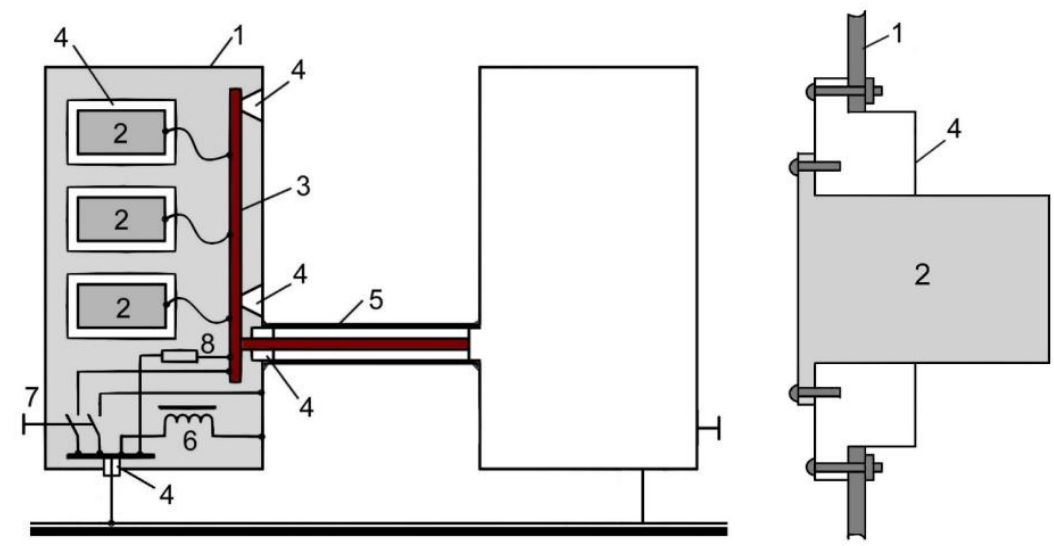

Fig4. Example of a grounding system "special floating ground".

1 - metal structure of the cabinet; 2 - electronic device, e.g. DPR, 3 - copper bus establishing the zero potential area; 4 - insulators; 5 - screening enclosure; 6 - choke; 7 - contact breaker; 8 - high-voltage high-resistance resistor.

I suggest that a solution is to use a special (advanced) floating ground, i.e. the local area of reference zero potential (local ground), which is established by, for example, a special copper bus 3, resting on insulators in a metal cabinet 1 with the electronic equipment 2 inside, Fig. 3. Separate bodies of each electronic device 2, insulated (with minimum capacitance from the cabinet) by means of insulators 4 should be connected to a copper bus 3. Copper buses of different cabinets located close to each other in one room should be connected to an external copper bus located coaxially in a shielded enclosure 5 , Fig. 4.

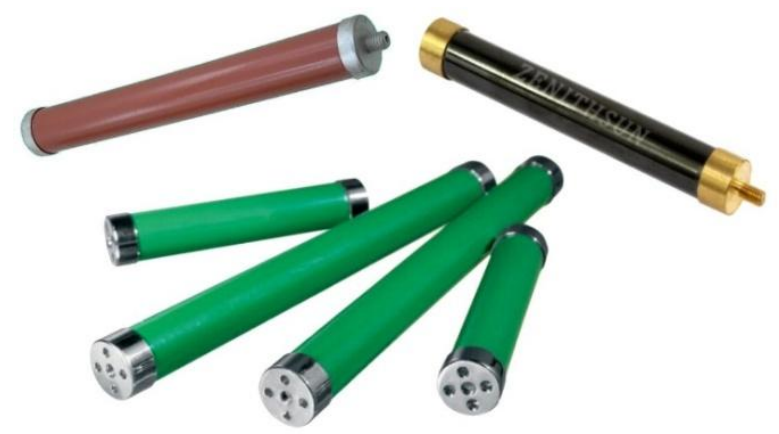

Fig5. High-voltage high-resistance resistors 
The zero potential local area should be connected to the external grounding system only via a highvoltage high-resistance resistor 8 (Fig. 4), with the resistance rating of $100 \mathrm{MOhm}$ that prevents accumulation of static discharge on electronic device enclosures.

Between the distanced devices located in different rooms, the data exchange should be ensured via the optic fiber cable, while the discrete commands and the signals (such as ON-OFF)should be communicated through a special insulating interface (fast reed-switch relays with high-voltage inputoutput insulation -hercotrones), Fig. 6. [21].

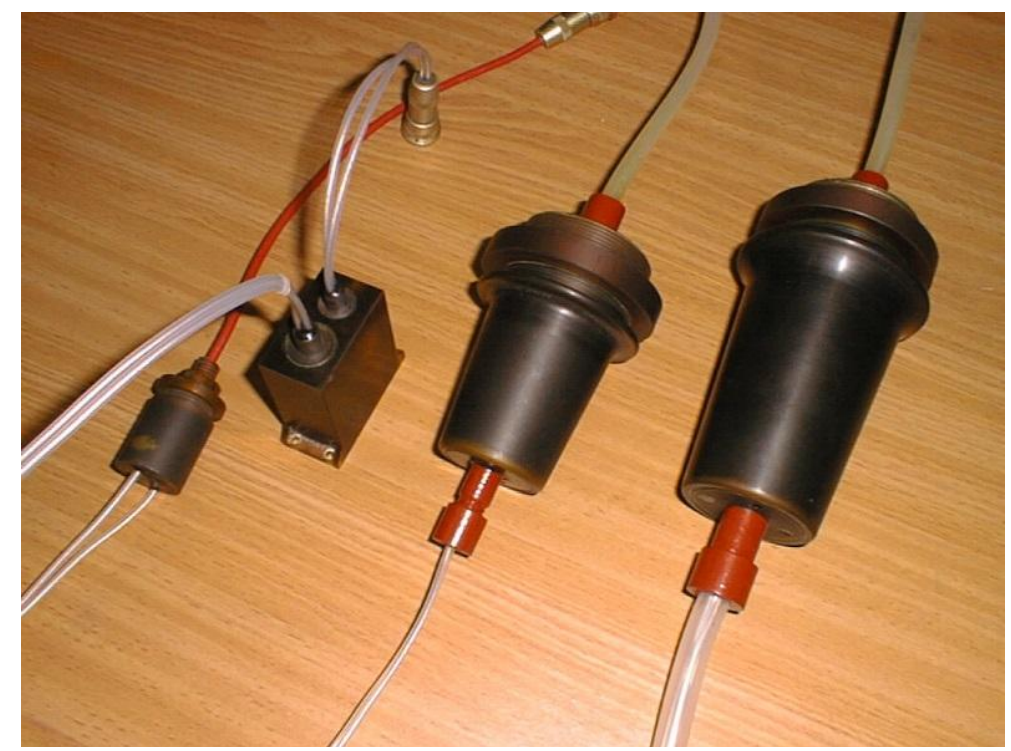

Fig6. High-voltage insulating interfaces (hercotrones) for operating voltage between input and output 10, 20, 50 and $70 \mathrm{kV}[21]$.

As mentioned above, modern electronic devices generally do not require functional grounding, so the solution offered above does not influence the operating performance of electronic equipment.

However, protection of personnel from a dangerous potential resulting from insulation damage upon the internal damage of equipment and cables inside the cabinet, was solved in the presented concept by other measures.

First, the metal cabinet can be grounded through a powerful high-frequency choke wrapped by a copper bus. Such a choke should have very low resistance under direct current and $50 \mathrm{~Hz}$ alternating current, but, at the same time, it should ensure high resistance under a lightning pulse and especially high resistance under a very short HEMP pulse, Fig. 7.
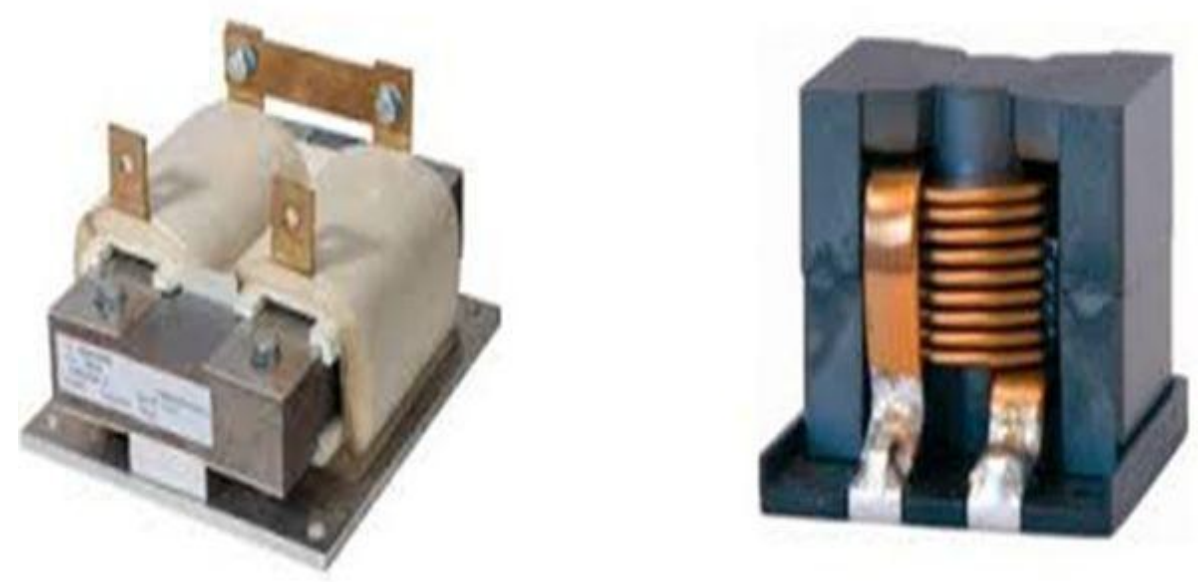

Fig. 7.Powerful high-voltage chokes

Second, the cabinet should be equipped with a 3-phase contact-breaker with high-power terminals and a handle, located outside the cabinet, Fig. 8. The contact-breaker should be closed when personnel are working inside the cabinet. 

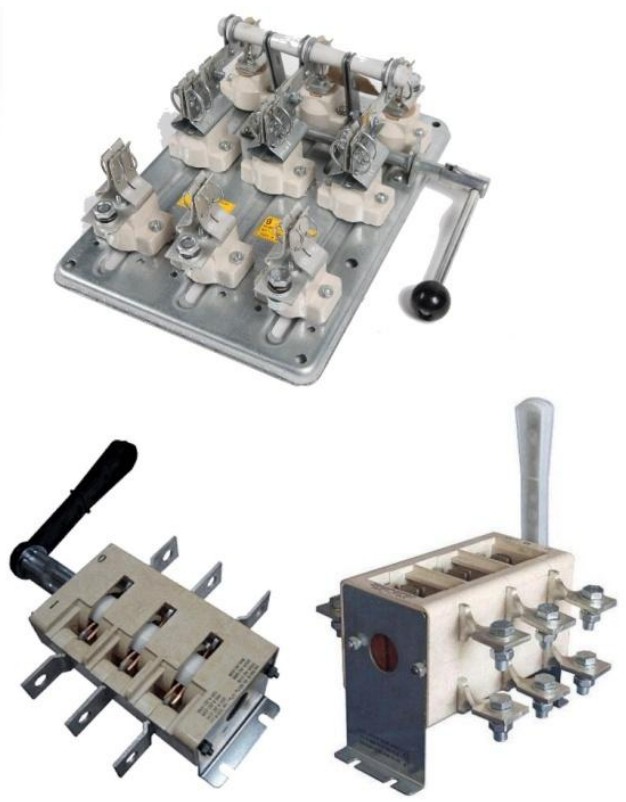

Fig. 8.High-power three-pole contact-breaker with a visible circuit disconnection

One terminal should be used to connect the internal insulated copper bus to the external grounding system; the second terminal provides direct connection between the cabinet and the external grounding system; the third is used to switch off the cabinet's door locking mechanism to ensure that the door can be opened only after the contact-breaker is closed. It is also possible to use a two-pole circuit breaker with additional auxiliary contact for these purposes.

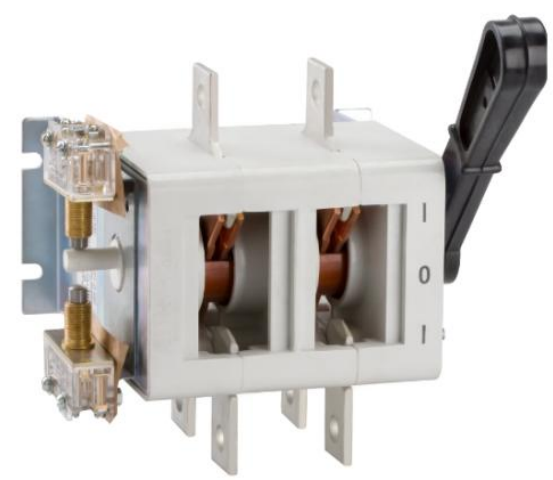

Fig. 9.Two-pole contact-breaker with auxiliary contact

Moreover, (if necessary) a temporary earth attached to the internal insulated copper bus can be used when someone is working inside the cabinet. However, I think it is not necessary.

\section{Conclusion}

The solution offered above rectifies the floating ground method issues. Thus, it can be widely used in the power industry to improve electronic equipment's resistance to powerful natural and intentional electromagnetic interferences.

\section{REFERENCES}

[1] Barreto R. M. EMC = Grounding on Automation and Control Systems. Applications to Eliminate Electromagnetic Interference in Industrial Plants. - Interference Technology, 2013, March 19, pp. $14-19$.

[2] Armstrong K. Planes Need to be Grounded to Something? - Interference Technology, July 1, 2013.

[3] Report ORNL/Sub/83-43374/2. Impact of Nominal Nuclear Electromagnetic Pulse on Electric Power Systems. - Oak Ridge National Laboratory, 1991, 94 p. 
[4] Duff W. G. Designing Electronic Systems for EMC: Grounding for the Control of EMI. Interference Technology, November 6, 2011.

[5] IEC 60364-7-707: 1984.Electrical installations of buildings - Requirements for special installations or locations - Earthing requirements for the installation of data processing equipment.

[6] Kuznetsov M. B. Protection against secondary appearances of a lightning and maintenance EMC of the equipment on sites of oil-and-gas branches. - Energoexpert, 2007, No. 2, pp. 61-65.

[7] Whitaker J. C. Electronic Systems Maintenance Handbook, Second Edition - CRC Press (Taylor \& Francis Group), Boca Raton - New York - London, 2001, 624 p.

[8] IEEE Std. 1100-2005. IEEE Recommended Practice for Powering and Grounding Electronic Equipment, 2005, $589 \mathrm{p}$.

[9] Gurevich V. Protection of Substation Critical Equipment Against Intentional Electromagnetic Threats. - Wiley, 2017, 228 p.

[10] Pamphlet No. 1110-3-2 Electromagnetic Pulse (EMP) and Tempest Protection for Facilities. Engineering and Design. Department of the Army, U.S. Army Corps of Engineers, 1990, 467 p.

[11] The Effects of Nuclear Weapons. - U. S. Department of Defense and Energy Research \& Development Administration, 1977, 660 p.

[12] AD-A009 228 Electromagnetic-Pulse Handbook for Electric Power Systems. Stanford Research Institute, 1975, $341 \mathrm{p}$.

[13] TM 5-690 Grounding and Bounding in Command, Control, Communications, Computer, Intelligence, Surveillance and Reconnaissance (C4ISR) Facilities. Headquarters Department of the Army, Washington, DC, 2002.

[14] Ricketts L. W., Bridges J. E., Miletta J. EMP Radiation and Protective Techniques. Wiley, New York - London - Sydney - Toronto, 1976, 380 p.

[15] Joffe E. B., Lock K. S. Grounds for Grounding. A circuit-to-System Handbook. - IEEE Press, Wiley, 2010, $1064 \mathrm{p}$.

[16] Report No. HDL-TM-81-9. Application of Filters for High-Altitude Electromagnetic Pulse Protection. - U. S. Army Electronics Research and Development Command, Harry Diamond Laboratories, 1981, $53 \mathrm{p}$.

[17] Longoria S. N. Facility Power Filters: Symmetric vs. Asymmetric Performance. - In Compliance, Sept. 2013, pp. $35-41$.

[18] Protection of High Voltage Power Network Control Electronics Against Intentional Electromagnetic Interferences (IEMI). Report WG C4.206 CIGRE, 2014, 27 p.

[19] MIL-HDBK-419A Grounding, Bonding, and Shielding for Electronic Equipment and Facilities, U. S. Department of Defense, 1987, 404 p.

[20] To Float or Not to Float? Analysis of a Floating vs. Grounded Output. Associated Power Technologies Inc. (www.aptsources.com/resources/pdf/Floating\%20Output.pdf).

[21] Gurevich V. Protection Devices and Systems for High-Voltage Applications. - Marcel Dekker, New York - Basel, 2003, 292 p.

\section{AUTHOR's BIOGRAPHY}

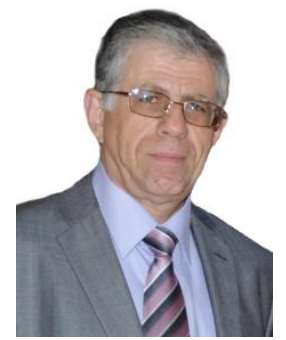

Vladimir I. Gurevich was born in Kharkov, Ukraine, in 1956. He received an M.S.E.E. degree (1978) at the Kharkov Technical University, named after P. Vasilenko, and a Ph.D. degree (1986) at Kharkov National Polytechnic University. His employment experience includes: teacher, assistant professor and associate professor at Kharkov Technical University, and chief engineer and director of Inventor, Ltd.

In 1994, he arrived in Israel and works today at Israel Electric Corp. as a senior specialist and Head of section of the Central Electric Laboratory.

$\mathrm{He}$ is the author of more than 200 professional papers and 13 books and holder of nearly 120 patents in the field of electrical engineering and power electronics. In 2006 he was Honorable Professor with the Kharkov Technical University. 